Revue d'histoire de l'Amérique française

ARS REVUE D.HISTOIRE DE L'AMÉRIQUE FRANÇAISE

\title{
La crise de la Marine française, d'après le Mémoire de Maurepas de 1745 sur la marine et le commerce
}

\section{Maurice Filion}

Volume 21, numéro 2, septembre 1967

URI : https://id.erudit.org/iderudit/302670ar

DOI : https://doi.org/10.7202/302670ar

Aller au sommaire du numéro

Éditeur(s)

Institut d'histoire de l'Amérique française

ISSN

0035-2357 (imprimé)

1492-1383 (numérique)

Découvrir la revue

Citer cet article

Filion, M. (1967). La crise de la Marine française, d'après le Mémoire de Maurepas de 1745 sur la marine et le commerce. Revue d'histoire de l'Amérique française, 21(2), 230-242. https://doi.org/10.7202/302670ar d'utilisation que vous pouvez consulter en ligne. 


\section{LA CRISE DE LA MARINE FRANÇAISE, D'APRĖS LE MÉMOIRE DE MAUREPAS DE 1745 SUR LA MARINE ET LE COMMERCE}

\section{I - INTRODUCTION}

La fin de l'aventure coloniale de la France en Amérique du Nord ne peut être un coup du destin subit comme une sonnerie de trompettes, c'est plutôt un drame longuement préparé dont la conquête de 1760 est l'épisole final avant la tombée du rideau. Acte brutal et précoce que cette rupture mais dont les causes à long terme sont discernables. Ainsi la crise de la Marine française dans la première partie du $18^{e}$ siècle permet d'éclairer plusieurs aspects de la décadence coloniale de la France en Amérique du Nord et laisse entrevoir l'acheminement de la Nouvelle-France vers son sort fatal. Un mémoire de Maurepas, écrit à la fin de $1745^{1}$, démontre sans ambages la nécessité des forces navales pour la France, fait état de l'importance du commerce maritime et colonial dans l'économie française et illustre la gravité de la crise de la Marine française à cette époque.

\section{II - LE MÉMOIRE DE MAUREPAS}

La Marine en France n'a pas toujours eu "le vent dans les voiles" malheureusement. C'est à Richelieu, "son véritable fondateur" 2, qu'il revient de lui avoir donné "le souffle initial", à Colbert, "son véritable créateur" 3 , le vent du large. Pour un si heureux départ, elle connut pourtant bien des avaries; et la pire, celle de son existence même mise en discussion: "Après la mort du Cardinal de Fleuri, on proposa de supprimer la Marine, comme on l'avait rejeté en 1681." " C'est pour en démontrer 189-224.

1 Archives Publiques du Canada, Archives de la Guerre, pièce 253,

2 Etienne Taillemite, Dictionnaire illustré de la Marine, 228.

3 Ibid., 55.

4 Archives publiques du Canada, Archives de la Guerre 253, 189. 
l'utilité et répondre aux accusations lancées par ses détracteurs que Maurepas écrivit au roi Louis $X V$, à la fin de 1745 , cet important mémoire.

Par bonheur, la Marine a un bon défenseur en la personne de Jean-Frédéric Phélipeau, comte de Maurepas. Celui-ci jouit à la fois de la tradition familiale des Pontchartrain qui ont servi la monarchie française sous Louis XIV et d'une expérience personnelle de 23 ans comme secrétaire d'État au département de la Marine et du Commerce, poste que le roi Louis XV lui avait confié en $1723^{5}$ et qu'il conservera jusqu'à sa disgrâce en 1749 . $\mathrm{Au}$ dire de l'armateur Fournier, "le Roy a un ministre à la tête de la marine qui ne cède en rien aux talents et aux connaissances de M. Colbert... il ne luy manque que de l'argent" ' Une longue expérience de plus de 50 ans dans le commerce maritime appuyait ce témoignage fort éloquent. L'étude du Mémoire de 1745 confirme cette opinion d'un contemporain de Maurepas, et révèle une personnalité différente de celle tombée en discrédit auprès de tant d'historiens. La largeur de vue sur les assises de la puissance française, l'examen en profondeur de la faiblesse de la marine, la démonstration évidente de l'insuffisance de fonds, tout le plaidoyer (car c'en est un) laisse voir les qualités de cet homme d'Etat et sa dramatique impuissance.

\section{LE ROLE DE LA MARINE}

Toute la défense de Maurepas en faveur de la marine repose sur un apophtegme d'ordre économique et politique: "LE COMMERCE FAIT LA RICHESSE ET CONSÉQUEMMENT LA PUISSANCE DES ETATS".7 Vérité indiscutable que le ministre de la Marine met en tête et bien en évidence du mémoire pour le rappeler à la réflexion du roi. Quel souverain ne désire pas la richesse, la puissance? Etre le plus riche, c'est être le plus puissant. C'est du coup attirer l'ambition dans un raisonnement et proposer une ligne d'action implacable: l'accroissement du

5 Lettre de M. Etienne Taillemite, 14 nov. 1964.

6 The Maurepas Papers, Catalogue No 2092 (Parke-Bernet), Galleries (New York, 1962), 32.

7 Archives publiques du Canada, Archives de la Guerre, 253, 190. 
commerce. Dans cette vue, le rôle imprescriptible de la marine est tout indiqué: "LES FORCES NAVALES sont absolument nécessaires pour le soutien du commerce maritime et pour la défense d'un État bordé par la Mer." ${ }^{8}$ La marine assume donc un rôle de premier plan, économique et militaire. Il reste à en démontrer la nécessité vitale dans les faits passés et présents de l'histoire.

Une première démonstration s'impose: l'importance du commerce maritime dans l'enrichissement d'une nation. Il y a d'abord le cabotage, commerce sans grands profits, pratiqué par tous les états maritimes pour leurs besoins, sans interdiction de la part des puissances maritimes, à la réserve qu'elles s'en sont approprié la meilleure part. Il y a surtout le grand commerce, celui de l'Amérique, de l'Afrique, des Indes Orientales. À l'égard de celui-là “qui donne le plus de profits, et qui procure les plus grandes richesses, les puissances maritimes se le sont réservé absolument". ${ }^{9}$ C'est une exclusivité péremptoire. Les petites nations ne font que parasiter ce commerce et encore par nécessité d'intérêts de la part des "grands". En ce domaine, point de partage. Même le puissant empereur Charles VI a dû supprimer, en l'an 1731, la compagnie d'Ostende et se retirer du commerce des Indes. Les Brésiliens ne sont les possesseurs tranquilles du Brésil que parce qu'ils ont permis aux Anglais de s'emparer du commerce et des richesses de ce pays. Seuls les Espagnols ont osé résister et leur résistance leur a valu la guerre avec les Anglais dont la cupidité, non satisfaite d'avoir enlevé à l'Espagne la Jamaïque, la Virginie et la plupart des autres colonies qu'ils possèdent et d'avoir obtenu le privilège de l" assiente" des Noirs et du vaisseau de permission, les a entraînés "à prétendre faire librement le reste du commerce, celui-là même qui par les lois d'Espagne est réservé aux Espagnols seuls". ${ }^{10}$

Ces prétentions cependant ne tiennent qu'à la supériorité sur mer des Anglais, supériorité accrue "à mesure que leurs for-

8 Ibid., 190.

9 Ibid., 191.

10 Ibid., 192. 
ces navales se sont développées, et que celles des autres États se sont affaiblies". 11 On ne saurait mieux souligner l'interdépendance entre la puissance des forces navales et la part du commerce maritime. Les graphiques suivants illustreront cette totale interdépendance.

"Les forces navales sont seules capables, en soutenant le commerce, de maintenir les Etats dans la possession de leurs revenus et conséquemment dans la conservation de leur puissance." ${ }^{12}$ L'importance du commerce maritime dans la puissance des Etats est en étroite relation avec la puissance de ses forces navales. On ne peut écarter l'un des aspects sans négliger l'autre. Grandeur politique et puissance maritime sont étroitement associées "dans le meilleur et dans le pire".

\section{LE COMMERCE MARITIME DE LA FRANCE}

À cette époque, le commerce maritime français est florissant. Il s'élève à plus de 300 millions de livres d'entrée et de sortie, en 1714. Les avantages en sont énormes. Il est, en effet, la cause principale du commerce intérieur et de la richesse des particuliers. La prospérité du pays repose sur le commerce maritime. Il assume la prospérité du pays: $1^{\circ}$ par le "mouvement prodigieux" et continuel d'entrée et de sortie de plus de 300 millions de marchandises et denrées tous les ans; $2^{\circ}$ par l'emploi qu'il procure à un nombre indéfini d'habitants; $3^{\circ}$ par l'afflux considérable d'or et d'argent qu'il apporte et par la circulation immense qu'il procure aux espèces monnayées; $4^{\circ}$ par les droits et impositions qu'il procure. On conçoit "facilement que c'est de ce commerce que dépendent les revenus du Roi, et que si par malheur il venait à cesser, l'on verrait bientôt les revenus diminuer à proportion de la diminution qu'il y aurait infailliblement dans le mouvement et la circulation qu'il occasionne". ${ }^{13}$ Autre avantage important: celui du change étranger "dont le cours est véritablement comme on dit, le Baromètre du commerce; toutes les fois que le change est au-dessus du

11 Ibid., 193.

12 Ibid., 193.

13 Ibid., 204. 
Graphique I

PARTAGE DU COMMERCE MARITIME

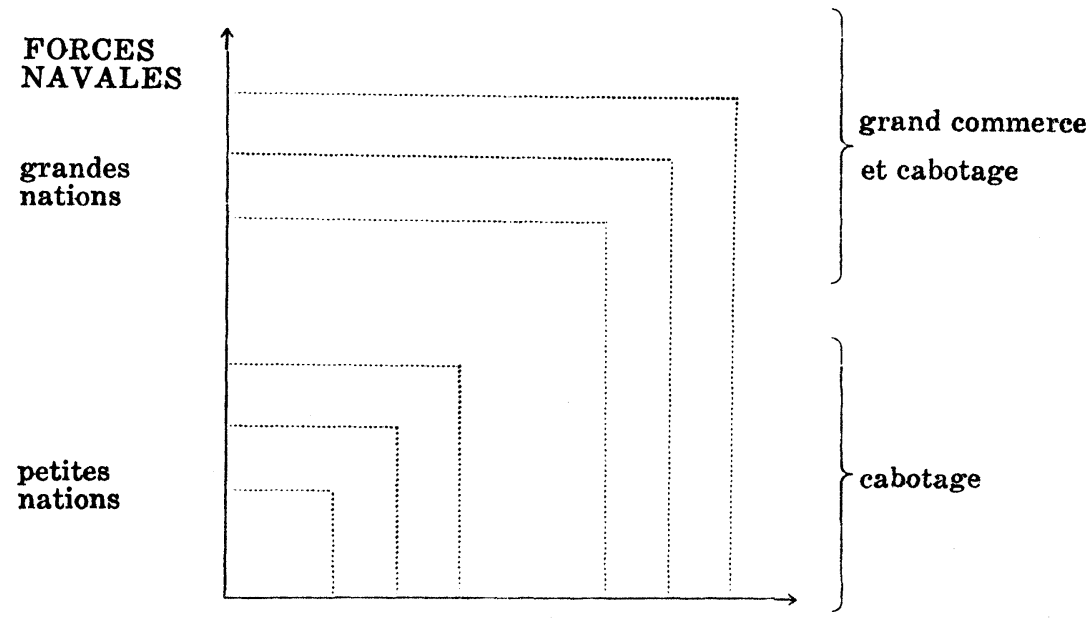

Graphique II

“PRÉTENTIONS” DES ÉTATS

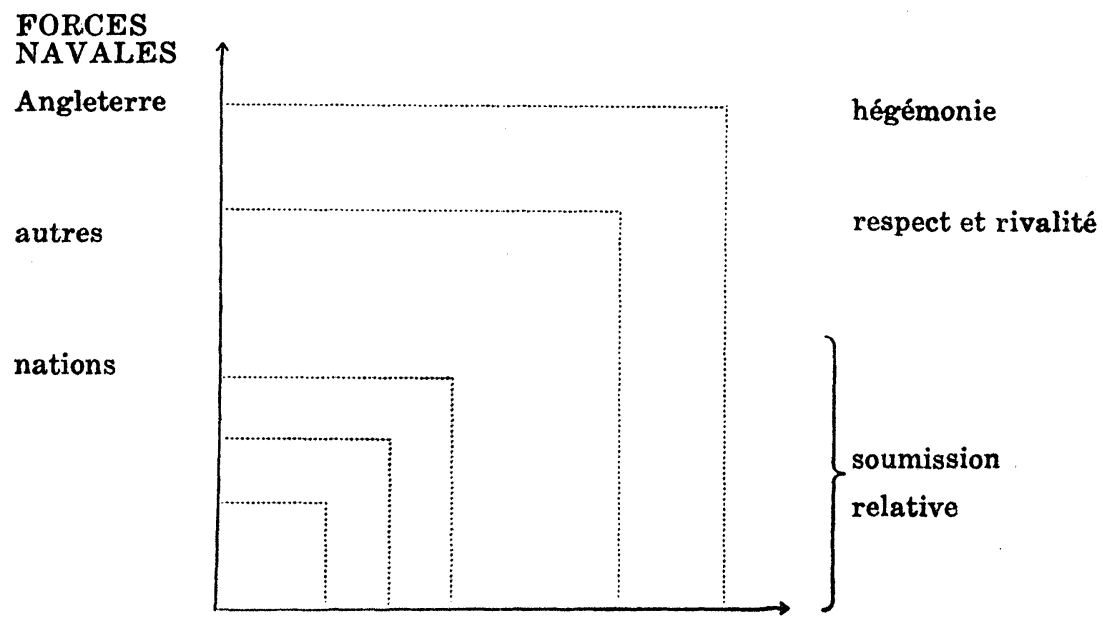


papier, c'est une preuve que l'étranger nous doit et que nous lui portons par conséquent plus de marchandise qu'il ne nous en fournit". ${ }^{14}$

Mais quel sera l'apport du commerce colonial à cette prospérité? Un tableau détaillé du commerce maritime nous le laisse entrevoir.

Tableau $I$

COMMERCE MARITIME DE LA FRANCE

Commerce avec les colonies françaises:

140 Millions

Commerce avec l'Espagne et les colonies espagnoles: $\quad 80 \mathrm{M}$.

Commerce du Levant et de Barbarie:

$30 \mathrm{M}$.

Commerce de la Cie des Indes en Orient

et à la Côte d'Afrique:

30 à $35 \mathrm{M}$.

Commerce avec l'Italie, le Portugal et les Etats du Nord: $20 \mathrm{M}$.

MONTANT GLOBAL:

$300 \mathrm{M}$.

Il convient de signaler que le commerce avec les colonies représente près de la moitié du commerce total, soit 140 millions sur 300 millions. C'est fausseté que de prétendre que les colonies coûtent cher et rapportent peu puisque le commerce colonial tient une place importante. D'ailleurs, le commerce semble bien orienté vers les colonies car les Cies du Levant et des Indes ne rapportent que 60 millions ou $20 \%$.

Autre constatation, le commerce avec l'Espagne et les colonies espagnoles est quatre fois supérieur au commerce avec les autres Etats européens. On le voit clairement, la France est liée économiquement à l'Espagne au moins aussi étroitement économiquement que politiquement. Il n'y a pas à s'étonner que la France se range aux côtés de l'Espagne durant la Guerre de la Succession d'Autriche. Le commerce avec les colonies, avec l'Espagne et ses colonies a la part du lion, soit 220 millions, ou près de $75 \%$. Enfin, il n'est fait aucune mention du commerce avec l'Angleterre ou avec ses colonies. Peut-on supposer qu'il soit quasi-inexistant en raison de la rivalité commerciale de ces deux puissances, malgré la politique de paix de l'abbé Dubois et du cardinal de Fleury?

14 Ibid., 205. 
Principale cause de la prospérité de la France, d'une infinité d'emplois, d'un afflux considérable d'or et d'argent, de l'abondance des revenus du roi, de l'avantage du change étranger, le commerce maritime est tributaire, dans une large mesure, du commerce colonial, soit $46.6 \%$. Les colonies constituent donc l'appoint principal de ce commerce si lucratif. C'est faire ressortir avantageusement l'utilité des colonies et apporter une réponse péremptoire à leurs détracteurs mal avisés. Une comparaison entre la croissance de la richesse de la France et l'augmentation du commerce colonial permet de faire ressortir avec évidence l'importance des colonies.

Tableau II

RICHESSE DE LA FRANCE (espèces monnayées)

vers 1650 :

100 millions

fin $d u 17^{e}$ s.: 500 millions

1745: 1200 millions
Tableau III

COMMERCE COLONIAL

DE LA FRANCE

(montant global d'entrée et de sortie)

règne de Louis XIV moins de 25 millions annuellement

guerre de succession 140 millions
d'Autriche: annuellement

"Bien inestimable" que le commerce avec les colonies "en ce qu'il fait vivre les uns, enrichit les autres et augmente les revenus du Roi. Il est constant qu'ils ont augmenté considérablement à mesure que le commerce des Colonies a augmenté, et que c'est par conséquent ce commerce qui est la principale cause de leur augmentation". ${ }^{15}$ Il importe fort de noter l'importance que Maurepas accorde aux colonies. On pourrait reprendre l'apophtegme initial et écrire: "Le commerce colonial fait la richesse et conséquemment la puissance des Etats." ${ }^{15} \mathrm{Ce}$ serait le plus bel hommage à l'esprit colonial de ce secrétaire d'État à la Marine et au Commerce maritime.

\section{LA CRISE DE LA MARINE FRANÇAISE}

C'est encore au commerce avec l'Amérique et les Indes que les Etats doivent l'établissement et l'accroissement de leurs 15 Ibid., 196. 
forces navales. Avant les grandes découvertes, il n'existait pas de marines nationales. Par la suite, l'établissement d'un nouveau commerce avec ces nouveaux pays et l'appropriation de ces nouveaux territoires déterminèrent la création de marines nationales. Portugais et Espagnols, possesseurs des plus grands établissements, eurent aussi les premiers le plus grand nombre de vaisseaux de force. Anglais et Hollandais, dans leur lutte contre l'Espagne, furent forcés d'entretenir plus de vaisseaux. Mais ce ne fut pour toutes les puissances, y compris la France, qu'au $17^{\mathrm{e}}$ siècle que la Marine eut une forme fixe pourvue d'une administration réglementée et d'une organisation permanente avec la construction des arsenaux et des grands ports, l'entretien d'un nombre réglé de vaisseaux de force et d'un corps d'officiers de Marine. ${ }^{16}$ Et toutes ces dépenses nouvelles proviennent de revenus nouveaux. "On jugera facilement que ce sont les revenus produits par ce commerce, qui ont fourni aux dépenses nécessaires pour les (forces navales) former et les entretenir, sans quoi il n'aurait pas été possible de pourvoir à d'aussi fortes dépenses." ${ }^{17}$ La Marine n'est donc pas une excroissance coûteuse du budget des États. Elle émarge en toute fierté, capable de pourvoir à ses propres investissements grâce aux revenus qu'elle apporte. Le commerce maritime pourvoit à ses propres investissements d'organisation et de croissance.

En France, la Marine tarde à apparaître. Si Richelieu lui a donné ses lettres de noblesses il faut attendre Colbert pour le grand départ, car ce n'est qu'en 1669 que la marine française eut un certain nombre de navires de force. Mais alors avec quelle vigueur la tâche est menée. La grandeur française est en voie de s'établir sur des assises solides: "production, marine, colonies et marchés". ${ }^{18}$ Mais jusques à quand cet effort ? Et dans quelle mesure ? Un tableau conparatif permettra d'apprécier l'ampleur des réalisations sous Louis XIV, le déclin et la faiblesse des tentatives qui suivirent.

16 Ibid., 208.

17 Ibid., 209.

18 A.T. Mahan, The Influence of Seapower Upon History, 62. 
FORCES NAVALES DE LA MARINE FRANÇAISE ET FONDS ORDINAIRES DE LA MARINE

Tableau IV

FORCES NAVALES DE LA

FRANCE

(1660-1715)

(nombre de vaisseaux

de force)

1660:
$1682:$
$1688:$
$1697:$
$1698:$
$1699:$
$1700:$
$1701:$
$1710:$
$1713:$
$1714:$
$1715:$

(1715-1745)

$\begin{array}{ll}1716: & 66 \\ 1719: & 49 \\ 1725: & 54 \text { (nombre réglé) } \\ 1740: & \\ 1744: & 54 \\ 1745: & 62\end{array}$
Tableau $V$

FONDS DE LA MARINE

FRANÇAISE

(1682-1715)

(millions de livres)

(fonds ordinaires et

extraordinaires)

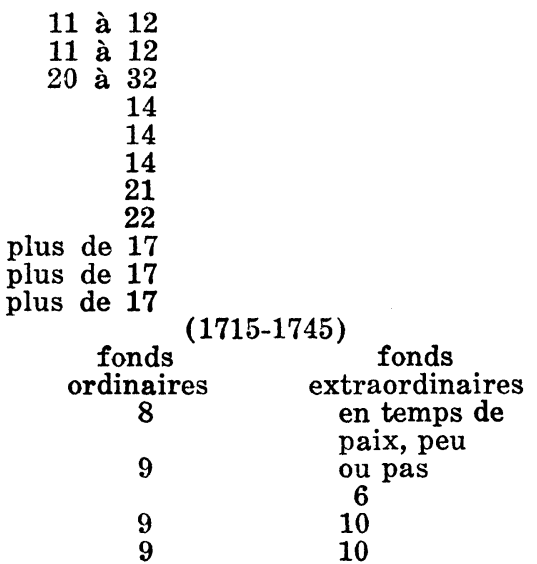

L'essor et la puissance de la Marine, sous Louis XIV, sont inscrits à la fois dans le nombre des navires de force et dans la somme des fonds ordinaires mis à sa disposition. Et cette puissance maritime, Colbert a réussi à l'établir en vingt ans seulement. Prodigieuse poussée de la nation française à la conquête de sa grandeur sur mer! Et durant 34 ans, Louis XIV maintient, coûte que coûte, une flotte de 120 navires. Il ne lésine pas sur les sommes affectées à la marine: les fonds extraordinaires oscillent entre 11 et 32 millions, selon les périodes de guerre ou de paix. La Marine tient une place importante dans la puissance française.

Mais tout s'étiole avec la fin du grand règne. La marine, en 1716, n'a que 66 navires et la plupart sont en mauvais état. Entre 1716 et 1745 , Louis XV n'entretiendra qu'une marine 
de force moyenne de 56 navires. C'est moins que la moitié de la force de la période précédente, 1682-1715, sans parler de l'état des navires. Au chapitre des fonds ordinaires, une somme moyenne de 8.8 millions est affectée à la marine, à comparer avec une moyenne de 18 millions pour la deuxième partie du règne de Louis XIV. Même en temps de paix, 1682-1688, 16991701,1715 , les fonds ordinaires n'ont été inférieurs à 11 millions, somme supérieure de 1 million aux fonds les plus élevés des années 1744 et 1745 , années de guerre cependant. La marine de guerre, après 1715, est délaissée, on le voit nettement par ces chiffres, laissée à pourrir. ${ }^{19}$

La diminution des fonds et des unités de combats atteste la gravité de la crise de la Marine française. En fait, elle est encore plus inquiétante que ne le laissent voir les chiffres précédents. Si encore cette force réduite était en bon état. Tant s'en faut. L'examen des fonds de la Marine accuse une situation plus déplorable encore et que Maurepas souligne au roi avec vigueur.

Tableau VI

RÉPARTITION DES FONDS ORDINAIRES DE LA MARINE 1716-1744 20

(millions de livres)

DÉPENISES FIXES ET INDISPENSABLES

Galères:

(1716-1719)

(1726-1744)

Colonies:

1.5

2.5

1.5

Appointements, soldes \&

hôpitaux, pensions:

DÉPENSES NON FIXES

(fluctuatives)

Entretien de la flotte

radoubs, constructions:

Investissements:

nil

1.6

TOTAL:

8.0

nil

9.0

19 Ibid., 65.

20 A.P.C., A.G., 211, 216. 
Tableau VII

AUGMENTATION PROCENTUELLE DU COUTT DES FOURNITURES NAVALES 1726-1744 21

Année:

Prix \%: 1726 $100 \%$

1744 $200 \%$

Il faut noter que dans un cas $50 \%$ et dans l'autre $44.4 \%$ des fonds ordinaires vont pour des dépenses "étrangères" à la marine: galères et colonies. On remarque alors que les dépenses fixes atteignent 7.4 millions; il ne reste qu'un maigre excédent de 600,000 pour l'entretien de la flotte, radoubs et constructions entre les années 1716-1719. Somme insuffisante même pour l'entretien de la flotte, et le nombre de navires tombe à 49 en 1719. Il n'est donc point question de constructions et "si dans la suite il en fut construit quelques-uns, ce ne fut qu'avec des fonds particuliers que l'on obtint de temps en temps et toujours difficilement pour ces constructions." ${ }^{22}$ C'est donc que les fonds ordinaires ne permettent aucuns investissements pour les navires et qu'il faut puiser ailleurs, à cette fin. Quand, en 1725 , le nombre de navires est réglé à 54, Maurepas fait voir au roi l'insuffisance de fonds pour un nombre pareil de navires et le roi consent à hausser les fonds de 1 million, ce qui laisse un excédent de moins de 1.6 million pour les dépenses qui ne sont pas fixes. Voilà qui est suffisant pour les premières années après 1726 et la flotte peut atteindre le nombre réglé de vaisseaux. Mais les fonds restent au même niveau durant 20 ans et durant ce temps le prix des fournitures double, en sorte que les fonds sont insuffisants même pour l'entretien à la fin de cette période. Mais cette hausse correspond à une demande de 20 millions de fonds extraordinaires, et ce sont deux années de guerre. Triste sort de la Marine! Il importe de préciser qu'il n'y avait pas de distinction avant 1716 entre fonds ordinaires et extraordinaires et que les sommes étaient "fixées en guerre comme en paix, tant pour les dépenses ordinaires que pour celles des armemens (sauf) qu'en tems de guerre elles étaient augmentées selon les dépenses occasionnées par la guerre

21 Ibid., 218.

22 A.P.C., A.G., 211. 
à la marine". ${ }^{23}$ Sous Louis XV, les fonds fixes de la Marine sont les fonds ordinaires; quant aux fonds "extraordinaires" pour les armements on décida d'en accorder au besoin seulement. Pratique infiniment préjudiciable à la Marine car en temps de paix on n'en accorde que peu ou pas selon les années et qu'en temps de guerre (depuis 1740) on ne tient pas compte des dépenses excédentes ou imprévues occasionnées par les événements. C'est la cause de l'endettement croissant de la marine.

Tableau VIII

1744 :

\begin{tabular}{cc}
\multicolumn{2}{c}{ DETTES DE LA MARINE 24 } \\
\multicolumn{2}{c}{ (millions de livres) } \\
fonds extraordinaires & dépenses \\
6 & 8 \\
10 &
\end{tabular}

On voit clairement que, malgré les fonds extraordinaires et les dettes de la marine, l'effort naval de la période 1726-1745 n'est pas comparable à celui du règne précédent. Même l'année la plus forte, soit 1745 avec 24 millions, est nettement inférieure au maximum de 32 millions de certaines années de la guerre de la Ligue d'Augsbourg, surtout si l'on songe que les coûts ont doublé en 1744! La France de Louis XV est chiche pour sa Marine.

\section{CONCLUSION}

Les démonstrations de Maurepas font ressortir avec évidence l'importance des forces navales. Non seulement la sécurité de la France est à ce prix, mais également sa prospérité et sa puissance. "Le Commerce fait la richesse et conséquemment la puissance des Etats." ${ }^{25}$ Mais dans cet enrichissement, la part du commerce colonial est prépondérante. L'augmentation de la richesse de la France va de pair avec l'augmentation du commerce colonial. Ce seul fait suffit à montrer l'importance primordiale des colonies. Les assises de la puissance française n'ont pas de plus ferme appui. Maurepas fait voir clairement

23 Ibid., 209.

24 Ibid., 218, 224.

25 Ibid., 190. 
la dépendance politique et économique de la métropole à l'égard des colonies et le rôle imprescriptible de la Marine pour leur soutien et leur conservation.

Il y aurait lieu de croire que de si fermes arguments trouveraient large audience. En fait ce sont ceux de rares isolés. Bien sûr, la Marine ne sera pas supprimée et Maurepas aura gain de cause sur ce point. Mais quant à redorer le blason de la Marine il s'y emploie inutilement depuis vingt-deux ans. Le "système politique" ${ }^{26}$ se désintéresse et de la Marine et des colonies. Après les largesses de Louis XIV, la mesquinerie sous Louis $\mathrm{XV}$, rien de plus. Sous prétexte de politique pacifiste, la Marine est sacrifiée à l'ambition de l'Angleterre. On lui ménage les fonds chichement. Maurepas a beau plaider sa cause, on lui refuse obstinément les secours nécessaires, même à la veille de la guerre. Depuis 1716 , c'est le même désintéressement, la même parcimonie. Face à l'aveuglement de la classe politique française, Maurepas fait preuve d'une rare clairvoyance, et le mémoire de 1745 nous révèle les qualités de vision d'un homme d'État beaucoup plus à la mesure de ces devanciers que l'Histoire ne nous l'a appris.

MAURICE FILION

Département d'Histoire

Université de Montréal

26 Cité par Roland Lamontagne, Aperçu structural du Canada au XVIII' siècle (Montréal, 1964), 60 . 